\title{
A meta-study on future work in information and communication technologies
}

\author{
Sercan Oruç ${ }^{1 *}$, Sencer Yeralan ${ }^{2}$ \\ ${ }^{1}$ Information Systems Department, Middle East Technical University, Turkey \\ ${ }^{2}$ Agricultural and Biological Engineering Department, University of Florida, USA
}

*Corresponding author: sercan.oruc@ gmail.com

(C) The Author(s)

2020.

Published by ARDA.

\begin{abstract}

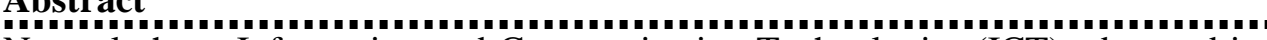
Not only have Information and Communication Technologies (ICT) advanced in rapid succession, but society has embraced these developments and implemented them in daily life. This adoption has not only affected our way of conducting routine activities, but also significantly modified the way we do things as well as the things we now do. At this junction, rather than how engineering should improve ICT, the appropriate question seems to be "what should ICT engineers focus on? This question involves not only the prediction of future trends, but core engineering ethics, since any new ICT development is likely to affect societal activity. We present a recent study where we developed an approach to predict future trends and use it to guide our research plans. In that sense, the current study is a meta study, that is, a study to reveal what and how we should conduct future studies.
\end{abstract}

Keywords: Internet and communication technology, technology trends, societal impact.

\section{Introduction}

Our intent is twofold. First, we would like to understand future trends in Information and Communications Technology (ICT) and how they affect societal conduct. Next, we would like to identify likely directions ICT will take as the various elements of the technology are either embraced or disregarded by society. The study is by nature inquisitive and argumentative. Our approach is to first survey available information, and then conduct extensive interviews to extract hints regarding future trends that may be better adopted by society.

The improvements in ICT have dramatic effects on how we live. These impacts are researched in various academic domains yet along mostly a reductionist viewpoint. There are many examples of research on the effects of ICT, for example, regarding education we cite, [1], [2], and [3] . Study [1] points to the increasing need for reliable translation services to accommodate the increase in networking among people of different languages and cultures. In that study, they experiment on the effect of translation technologies and machine translation systems on student performance and on the translation quality in classrooms. Their experimentation divides a group of students into two and compares the results of those two groups: one with, and the other without, ICT support. In study [2], they use the so-called Structure Equation Model (SEM) to understand the direct and indirect effects of instruction and the use of ICT on creating innovation according to the Socialization, Externalization, Combination, and Internalization (SECI) model. To quantify the study, they use questionnaires collecting opinions from 540 undergraduate students from three disciplines. In study [3], they measure the impacts of games on mobile devices on fifth-grade students' attitudes towards an "Information Technology and Software" course and on their understanding of programming concepts. The comparison of pre-test and post-test results for two separate test groups, they see an improvement in understanding, yet no change in attitude with the introduction of these so-called "mobile serious games". 
There are also studies focused on the relationship between ICT and urban structure. For example, study [4] explores the impact of ICT on urban structure. By using international data on city size distributions in various countries and country-level characteristics, the researchers test the effects of ICT. They report a correlation between the number of telephone lines per capita and the spatial dispersion of the population. In 2008 when this paper is published, they observe the evidence of internet usage to be more speculative, though they claim that it would have an even larger effect on urban structures.

In the environmental science domain, [5] points out that the relationship between ICT and environmental pollution from a spatial perspective is rarely studied. In this study, they use the panel data of 285 cities in China from 2003 to 2016 and spatial econometric models to see this effect. They see a positive spatial autocorrelation between these two.

In the e-commerce domain, in study [6] researchers interrogate the effect of continuous improvement of ICT applications on e-customer behavior. They conduct experimental studies on social media users to see how improvements in ICT applications lead to better e-customer behavior.

From the perspective of health and ICT, study [7] states that there are only a few studies conducted on the relationship between headaches and computer usage. To contribute to this area, 2216 IT staff are studied to uncover potential factors leading to headache disorders. Data is collected via questionnaires and processed using statistical methods.

In the domain of economics, study [8] develops and shares productivity frameworks and models to measure the ICT cybersecurity negative externalities spillover effects on sustainable economic growth. The study treats ICT cybersecurity's negative externality spillover effects similarly with negative externalities generated by pollutants' emissions.

In addition to these domains, in organizational science, ICT evolution and the effect of it on firms is well documented [9]. Although this is the case, whether the pace of research on the effect of technology to everyday life can cope with the rapid changes in the industry is questionable. There are also researches which starts creating taxonomy of personal processes [10] and developing ICT solutions to manage those processes which emerge in everyday life [11].

In this paper, we first share a research on the news around IT industry and then share an idea on how to approach studying the effects of ICT on everyday life.

Structurally, Section 2 gives information on material and methods, Section 3 provides the background of the study, Section 4 shares the results and discussions whereas Section 5 gives our conclusions.

\section{Material and methods}

We chose 10 random days in a one year period between July 1, 2019 and December 31, 2019, and went through a total of 951 Hacker News (https://news.ycombinator.com/) stories. Hacker News is arguably one of the best news resources in the intersection of technology and business. Its guidelines [12] list two main rules for what to have as news and what not:

- "On-Topic: Anything that good hackers would find interesting. That includes more than hacking and startups. If you had to reduce it to a sentence, the answer might be: anything that gratifies one's intellectual curiosity." 
- "Off-Topic: Most stories about politics, or crime, or sports, unless they're evidence of some interesting new phenomenon. Videos of pratfalls or disasters, or cute animal pictures. If they'd cover it on TV news, it's probably off-topic."

Those 10 random days and number of news published in Hacker News are listed in

Table 1.

Table 1. Random 10 days within six months and number of news posted in corresponding days

\begin{tabular}{|c|c|c|c|c|c|}
\hline Day & Date & $\begin{array}{c}\text { \# of } \\
\text { News }\end{array}$ & Day & Date & $\begin{array}{c}\text { \# of } \\
\text { News }\end{array}$ \\
\hline 9 & $2019-07-09$ & 96 & 95 & $2019-10-03$ & 97 \\
\hline 26 & $2019-07-26$ & 83 & 122 & $2019-10-30$ & 100 \\
\hline 50 & $2019-08-19$ & 101 & 152 & $2019-11-29$ & 79 \\
\hline 65 & $2019-09-03$ & 102 & 165 & $2019-12-12$ & 84 \\
\hline 78 & $2019-09-16$ & 110 & 183 & $2019-12-30$ & 99 \\
\hline
\end{tabular}

Among those news samples, we picked the ones that gave information on topics that could have a direct impact on people's personal lives. In the first step of this selection, we went through the headers of those 951 news items. 78 of those news posts are included in the second phase of the selection. In the second phase, we read those 78 different hacker news posts and included the ones relevant to our topic for the final analysis.

The inclusion criteria for a news post is as follows:

- Post should have ICT related content

- The content of the post should be directly related with the individuals' daily lives.

\section{Theory}

There are various studies which suggest ways to evaluate social impacts of ICTs.

With study [13] researchers approach this from a "quality of life" perspective to evaluate the social impacts of ICTs. They conduct two sets of telephone surveys with 11 months in between and analyze the quality of life change as well as ICT access and e-services usage change. Then, they check the correlation between those. They conclude that the effect of ICT on social capital exists although the causal relations among changes of ICT usage, access, and social capital is not exactly conclusive.

In study [14], researchers criticize the domination of market-oriented, functionalist, and instrumental views by "suggesting a critical neo-humanist alternative". In their paper they give Habermasian lifeworld-in-system with nine structural segments and argue that by investigating the influence of ICT on the process of communicative action process, those segments can be evaluated.

Another different approach to the social impact of ICT is from political philosophy. Study [15] analyzes how the existence and higher usage of ICT facilitate the realization of New Social Movements by allowing visibility, scope, and circulation.

In another study [16], researchers share a new index with name "Gross Social Feel-good" (GSF) index that can be used in the evaluation of environmental, social and economic influences of ICT services on sustainable 
development. They also propose a method for evaluating the happiness index via "questionnaires about the necessity, usability and impression of ICT services".

\section{Results and discussion}

After choosing 78 hacker news posts in the first phase, with the information gathered by detailed reading, a portion of them were excluded in the second phase. Among those not-excluded hacker news posts, 37 of them are grouped below, together with some descriptions.

Data collected in social platforms, applications, or even authorities are not always used in the way the data owners accept it to be.

- "Brazil fines Facebook \$1.6 million for improper sharing of user data" [17]

- "Facebook Will Now Use Oculus VR Data for Ads Just as Everyone Expected" [18]

- "The UK authorities made illegal copies of the \#Schengen Information System, incl photos \& fingerprints of EU citizens and gave access to US companies" [19]

- “Google Is Evil" [20]

Although accessed data is used in multi-varied ways, it is not easy to reach data collected by other parties.

- "Victory! EFF Wins Access to License Plate Reader Data to Study How Law Enforcement Uses the Privacy Invasive Technology" [21]

The way citizens behave are sometimes forced by the government by policies and legal acts.

- "Greeks set to face heavy fines if they don't spend 30 per cent of their income electronically." [22]

- "Hong Kong protests: Authorities to announce face mask ban" [23]

- "Hong Kong's protesters put AirDrop to ingenious use to breach China's Firewall" [24]

- "Getting a new mobile number in China will involve a facial-recognition test" [25]

Governments even sometimes shutdown the Internet to create repression or watch the citizens closely with surveillance technologies or intervene the decisions of big tech companies.

- "Life in an Internet Shutdown: Crossing Borders for Email and Contraband SIM Cards" [26]

- "Surveillance camera statistics: which cities have the most CCTV cameras?" [27]

- "Australia's anti-encryption laws being used to bypass journalist protections, expert says" [28]

- Attorney General Bill Barr Will Ask Zuckerberg To Halt Plans For End-To-End Encryption Across Facebook's Apps [29]

Sometimes, the decisions made by giant tech companies or companies having many people's personal data are found to be objectionable by data owners.

- "Google contractors reportedly targeted homeless people for Pixel 4 facial recognition" [30]

- "Here's that hippie, pro-privacy, pro-freedom Apple y'all so love: Hong Kong protest safety app banned from iOS store" [31]

- "My GitHub account has been restricted due to US sanctions as I live in Crimea." [32]

- "GitHub blocked my account and they think I'm developing nuclear weapons" [33]

- “Amazon's Next-Day Delivery Has Brought Chaos And Carnage To America's Streets - But The World's Biggest Retailer Has A System To Escape The Blame" [34]

- "Kaspersky in the Middle - what could possibly go wrong?" [35]

Sometimes those giant tech companies make decisions to minimize legal risks or protect their ecosystem.

- "Exclusive: Fearing data privacy issues, Google cuts some Android phone data for wireless carriers" [36] 
- "Twitter will not accept advertising from state-controlled news media entities." [37]

- Today's Firefox Blocks Third-Party Tracking Cookies and Cryptomining by Default [38]

There is an ongoing war between malicious hackers and ethical hackers.

- "Dutch police take down hornets' nest of DDoS botnets" [39]

- "Terrorists Turn to Bitcoin for Funding, and They're Learning Fast" [40]

- "Indian nuclear power plant's network was hacked, officials confirm" [41]

- "Takeaways from the $\$ 566 \mathrm{M}$ BriansClub breach" [42]

- "Statement: Intention to fine Marriott International, Inc more than $£ 99$ million under GDPR for data breach" [43]

- Unprotected Elasticsearch database exposes sensitive information of over 20 million Ecuador citizens [44]

Meanwhile, companies try to patch every gap they have in their software systems against vulnerabilities.

- “Zoom Zero Day: 4+ Million Webcams \& maybe an RCE? Just get them to visit your website!" [45]

- "The July 2019 Security Update Review" [46]

Machine Learning and Deep Learning technologies (i.e. per-trained models) are now readily available. These technologies facilitate various new implementations, some generally viewed as good and some not.

- “Fraudsters deepfake CEO’s voice to trick manager into transferring \$243,000” [47]

- "Face Recognition Using Pytorch" [48]

- "DeepFaceLab" [49]

- "Beijing Internet Court launches online litigation service center" [50]

- "Lixil AI-based Toilet Analyzes Shape \& Size of Feces with Camera and LEDs" [51]

ICT companies are creating new business models in various industries with the promise of improving or expanding the business of existing companies. Some of these new business models do not yield the intended results.

- “India’s Restaurants Rebel Against Food Delivery Apps" [52]

ICT also have impact on the behavior of individuals.

- "Survey shows average person grows frustrated after waiting 16 seconds for a webpage to load, 25 seconds for traffic signal to change." [53]

\section{Conclusion}

Our survey of on-line resources as well as our deliberations with people from different walks of life lead us to construct the following list of fundamental elements.

1. As mobile devices are becoming more and more ubiquitous, they are considered to be a permanent feature of any future trend.

2. As connectivity improves, computing power will be independent of the access device. That is, not all data processing needs to be on a mobile device, but rather may be seamlessly shifted to servers or other cloud facilities.

3. The so-called "smart" devices provide information when needed. Future devices are expected to go beyond the smarts to display rudimentary traits of consciousness. As such, these personal devices will provide unsolicited guidance and advice, make suggestions without the user consulting the device.

4. Extreme customization, not unlike friends or a person and a pet, seems to be a feature that is not only likely. But one that will also be embraced by society. 
These findings lead to the notion that the further integration of mobile devices will result in more personal, more customized, and more peer-to-peer-like platforms. If a device that is quite familiar with the needs, character, and whims of a user is to provide unsolicited input, society would be accepting the proposition that the more routine aspects of daily life be delegated to these devices. A bit like the old alarm clock that helps with a good night's sleep by elevating the need to keep checking the time, these personal devices may assist in routine tasks, usually by prompting the user to take action.

Here, we see a parallel to Business Process Management (BPM) systems. These systems track the activities of a business and prompt the system to take action at critical times. Moreover, they keep track of the performance of the system and help to identify possible aspects that may be improved. Similarly, mobile devices that act as a Personal Process Management (PPM) may be expected to be welcome by society in the near future. These PPM systems may prompt the user to help meet deadlines, pay bills, renew licenses, remind events, as some smart calendars now do. Moreover, these systems may also keep track of the user, suggest taking breaks, seeing a medical specialist, or eat certain foods that they may have neglected. Beyond physical help, tracking the mood of the user, PPM systems may engage in conversation or surprise the user when it seems beneficial for the psychological well-being of the user.

Our meta-study begins with an investigation into the likely directions ICT will take and concludes with one possible likely mega-trend, namely PPM. This paper presents the rational behind this identification and provides a methodology that may also be used to predict future trends in other industries that have a great impact on society, such as technologies that deal with the environment, security and privacy, and healthcare.

\section{References}

[1]Y. A. Gomaa, R. Aburaya, and A. Omar, "The effects of information technology and e-learning systems on translation pedagogy and productivity of EFL learners," in 2019 International Conference on Innovation and Intelligence for Informatics, Computing, and Technologies, 3ICT 2019, 2019.

[2]N. Songkram and S. Chootongchai, "Effects of pedagogy and information technology utilization on innovation creation by SECI model," Educ. Inf. Technol., 2020.

[3]M. Yallihep and B. Kutlu, "Mobile serious games: Effects on students' understanding of programming concepts and attitudes towards information technology," Educ. Inf. Technol., vol. 25, no. 2, pp. 1237 1254, Mar. 2020.

[4]Y. M. Ioannides, H. G. Overman, E. Rossi-Hansberg, and K. Schmidheiny, "The effect of information and communication technologies on urban structure," in Economic Policy, 2008, vol. 23, no. 54, pp. 201-242.

[5]Z. Cheng, L. Li, and J. Liu, "The effect of information technology on environmental pollution in China," Environ. Sci. Pollut. Res., vol. 26, no. 32, pp. 33109-33124, Nov. 2019.

[6]A. A. Meteab, A. S. Sadiq, and H. K. Hadrawi, "Effect of continuous improvement of information technology applications on E-costumer behavior in social media," Webology, vol. 17, no. 1, 2020.

[7]C. Li et al., "Prevalence of primary headache disorders among information technology staff in China: The negative effects of computer use and other correlative factors," BMC Public Health, vol. 20, no. 1, Apr. 2020.

[8]E. M. Ahmed, "Modelling Information and Communications Technology Cyber Security Externalities Spillover Effects on Sustainable Economic Growth,” J. Knowl. Econ., 2020.

[9]Kuo-Hao Lee, "The Advancement of Information Technology and Its Effect on Industries," Bus. Manag. Dyn., vol. 9, no. 8, pp. 1-6, 2020.

[10] S. Oruç, P. E. Eren, A. Koçyiğit, and S. Yeralan, “A Taxonomy for Personal Processes: Results from a Semi-structured Interview," Springer, Cham, 2019, pp. 771-782.

[11] S. Oruc, P. E. Eren, and A. Kocyigit, “A Reference Model for Personal Process Management (PPM) 
Systems," in Iberian Conference on Information Systems and Technologies, CISTI, 2019, vol. 2019June.

[12] "Hacker News Guidelines.” [Online]. Available: https://news.ycombinator.com/newsguidelines.html. [Accessed: 05-Jul-2020].

[13] Y. C. You and S.-F. Tseng, "Another Way to Evaluate Social Impacts of ICTs in Taiwan: A Quality of Life Perspective," in 101st Annual Meeting of the American Sociological Association, 2006.

[14] D. O'Donnell and L. B. Henriksen, "Philosophical foundations for a critical evaluation of the social impact of ICT,” J. Inf. Technol., vol. 17, no. 2, pp. 89-99, Jun. 2002.

[15] F. F. Morador and J. C. Vásquez, "New social movements, the use of ICTs, and their social impact," Rev. Lat. Comun. Soc., vol. 71, pp. 398-412, 2016.

[16] M. Tsuda et al., "New index for social impact assessment of ICT services," in IEEE International Symposium on Electronics and the Environment, 2007, pp. 16-18.

[17] R. Brito, "Brazil fines Facebook \$1.6 million for improper sharing of user data - Reuters," 2019. [Online]. Available: https://www.reuters.com/article/us-facebook-brazil-fine/brazil-fines-facebook-1-6million-for-improper-sharing-of-user-data-idUSKBN1 YY0VK. [Accessed: 21-Jul-2020].

[18] J. Hruska, "Facebook Will Now Use Oculus VR Data for Ads Just as Everyone Expected ExtremeTech," 2019. [Online]. Available: https://www.extremetech.com/gaming/303310-facebookoculus-vr-data-advertising. [Accessed: 21-Jul-2020].

[19] S. in 't Veld, ""The UK authorities made illegal copies of the \#Schengen Information System, incl photos \&amp; fingerprints of EU citizens and gave access to US companies $\square$ Will the @EU_Commission present findings of a detailed \&amp; swift inquiry to @Europarl_EN? Questi." [Online]. Available: https://twitter.com/SophieintVeld/status/1154689216170926080. [Accessed: 21Jul-2020].

[20] J. Anomaly, “Google Is Evil - Fake Nous," 2019. [Online]. Available: https://fakenous.net/?p=742. [Accessed: 21-Jul-2020].

[21] J. Lynch, "Victory! EFF Wins Access to License Plate Reader Data to Study How Law Enforcement Uses the Privacy Invasive Technology | Electronic Frontier Foundation," 2019. [Online]. Available: https://www.eff.org/press/releases/victory-eff-wins-access-license-plate-reader-data-study-how-lawenforcement-uses. [Accessed: 21-Jul-2020].

[22] T. Rees, "Greeks set to face heavy fines if they don't spend 30 per cent of their income electronically," 2019. [Online]. Available: https://www.smh.com.au/business/markets/greeks-set-toface-heavy-fines-if-they-don-t-spend-30-per-cent-of-their-income-electronically-20191209p53i14.html. [Accessed: 21-Jul-2020].

[23] "Hong Kong protests: Authorities to announce face mask ban - BBC News," 2019. [Online]. Available: https://www.bbc.com/news/world-asia-china-49918889. [Accessed: 21-Jul-2020].

[24] M. Hui, "Hong Kong protesters use AirDrop to breach China's Firewall — Quartz," 2019. [Online]. Available: $\quad$ https://qz.com/1660460/hong-kong-protesters-use-airdrop-to-breach-chinas-firewall/. [Accessed: 21-Jul-2020].

[25] J. Li, "China introduces facial-recognition step to get new mobile number - Quartz," 2019. [Online]. Available: $\quad$ https://qz.com/1720832/china-introduces-facial-recognition-step-to-get-new-mobilenumber/. [Accessed: 21-Jul-2020].

[26] P. Kingsley, "Life in an Internet Shutdown: Crossing Borders for Email and Contraband SIM Cards The New York Times,” $2019 . \quad$ [Online]. Available: https://www.nytimes.com/2019/09/02/world/africa/internet-shutdown-economy.html. [Accessed: 21Jul-2020].

[27] P. Bischoff, "Surveillance Camera Statistics: Which City has the Most CCTV Cameras?," 2019. [Online]. Available: https://www.comparitech.com/vpn-privacy/the-worlds-most-surveilled-cities/. 
[Accessed: 21-Jul-2020].

[28] J. Taylor, "Australia's anti-encryption laws being used to bypass journalist protections, expert says | Australia news | The Guardian," 2019. [Online]. Available: https://www.theguardian.com/australianews/2019/jul/08/australias-anti-encryption-laws-being-used-to-bypass-journalist-protections-expertsays. [Accessed: 21-Jul-2020].

[29] R. Mac and J. Bernstein, "Attorney General Bill Barr Will Ask Zuckerberg To Halt Plans For EndTo-End Encryption Across Facebook's Apps," 2019. [Online]. Available: https://www.buzzfeednews.com/article/ryanmac/bill-barr-facebook-letter-halt-encryption. [Accessed: 21-Jul-2020].

[30] S. Hollister, "Google contractors reportedly targeted homeless people for Pixel 4 facial recognition The Verge," 2019. [Online]. Available: https://www.theverge.com/2019/10/2/20896181/googlecontractor-reportedly-targeted-homeless-people-for-pixel-4-facial-recognition. [Accessed: 21-Jul-2020].

[31] K. McCarthy, "Here's that hippie, pro-privacy, pro-freedom Apple y'all so love: Hong Kong protest safety app banned from iOS store - The Register," 2019. [Online]. Available: https://www.theregister.com/2019/10/02/apple_hong_kong/. [Accessed: 21-Jul-2020].

[32] A. Kashkin, "Project status · Issue \#289 · tkashkin/GameHub · GitHub," 2019. [Online]. Available: https://github.com/tkashkin/GameHub/issues/289. [Accessed: 21-Jul-2020].

[33] H. Saeedi, "GitHub blocked my account and they think I'm developing nuclear weapons $\mid$ by Hamed Medium,” 2019. [Online]. Available: https://medium.com/@hamed/github-blocked-my-account-andthey-think-im-developing-nuclear-weapons-e7e1fe62cb74. [Accessed: 21-Jul-2020].

[34] C. O’Donovan and K. Bensinger, “Amazon's Next-Day Delivery Has Brought Chaos And Carnage To America's Streets - But The World's Biggest Retailer Has A System To Escape The Blame,” 2019. [Online]. Available: https://www.buzzfeednews.com/article/carolineodonovan/amazon-next-daydelivery-deaths. [Accessed: 21-Jul-2020].

[35] W. Palant, "Kaspersky in the Middle - what could possibly go wrong? | Almost Secure," 2019. [Online]. Available: https://palant.info/2019/08/19/kaspersky-in-the-middle-what-could-possibly-gowrong/. [Accessed: 21-Jul-2020].

[36] A. Moon and P. Dave, "Exclusive: Fearing data privacy issues, Google cuts some Android phone data for wireless carriers - Reuters," 2019. [Online]. Available: https://www.reuters.com/article/us-alphabetdata-exclusive-idUSKCN1V90SQ. [Accessed: 21-Jul-2020].

[37] Twitter, "Updating our advertising policies on state media," 2019. [Online]. Available: https://blog.twitter.com/en_us/topics/company/2019/advertising_policies_on_state_media.html. [Accessed: 21-Jul-2020].

[38] M. Wood, "Today's Firefox Blocks Third-Party Tracking Cookies and Cryptomining by Default - The Mozilla Blog,” 2019. [Online]. Available: https://blog.mozilla.org/blog/2019/09/03/todays-firefoxblocks-third-party-tracking-cookies-and-cryptomining-by-default/. [Accessed: 21-Jul-2020].

[39] C. Cimpanu, "Dutch police take down hornets' nest of DDoS botnets | ZDNet," 2019. [Online]. Available: https://www.zdnet.com/article/dutch-police-take-down-hornets-nest-of-ddos-botnets/.

[40] N. Popper, "Terrorists Turn to Bitcoin for Funding, and They're Learning Fast - The New York Times,” 2019. [Online]. Available: https://www.nytimes.com/2019/08/18/technology/terroristsbitcoin.html. [Accessed: 21-Jul-2020].

[41] S. Gallager, "Indian nuclear power plant's network was hacked, officials confirm | Ars Technica," 2019. [Online]. Available: https://arstechnica.com/information-technology/2019/10/indian-nuclearpower-company-confirms-north-korean-malware-attack/. [Accessed: 21-Jul-2020].

[42] B. Krebs, "Takeaways from the $\$ 566 \mathrm{M}$ BriansClub breach - Krebs on Security," 2019. [Online]. Available: https://krebsonsecurity.com/2019/10/takeaways-from-the-566m-briansclub-breach/. [Accessed: 21-Jul-2020].

[43] UK ICO, "Statement: Intention to fine Marriott International, Inc more than $£ 99$ million under GDPR 
for data breach | ICO," 09-Jul-2019. [Online]. Available: https://ico.org.uk/about-the-ico/news-andevents/news-and-blogs/2019/07/statement-intention-to-fine-marriott-international-inc-more-than-99million-under-gdpr-for-data-breach/. [Accessed: 21-Jul-2020].

[44] "Unprotected Elasticsearch database exposes sensitive information of over 20 million Ecuador citizens | Cyware Alerts - Hacker News," 2019. [Online]. Available: https://cyware.com/news/unprotected-elasticsearch-database-exposes-sensitive-information-of-over-20million-ecuador-citizens-58e5add8/. [Accessed: 21-Jul-2020].

[45] J. Leitschuh, "Zoom Zero Day: 4+ Million Webcams \& maybe an RCE? Just get them to visit your website! | by Jonathan Leitschuh | InfoSec Write-ups | Medium," 08-Jul-2019. [Online]. Available: https://medium.com/bugbountywriteup/zoom-zero-day-4-million-webcams-maybe-an-rce-just-getthem-to-visit-your-website-ac75c83f4ef5. [Accessed: 21-Jul-2020].

[46] D. Childs, "Zero Day Initiative — The July 2019 Security Update Review," 2019. [Online]. Available: https://www.zerodayinitiative.com/blog/2019/7/9/the-july-2019-security-update-review. [Accessed: 21-Jul-2020].

[47] R. Lakshmanan, "Fraudsters deepfake CEO's voice to trick manager into transferring \$243,000." [Online]. Available: https://thenextweb.com/security/2019/09/02/fraudsters-deepfake-ceos-voice-totrick-manager-into-transferring-243000/. [Accessed: 21-Jul-2020].

[48] T. Esler, "GitHub - timesler/facenet-pytorch: Pretrained Pytorch face detection (MTCNN) and recognition (InceptionResnet) models." [Online]. Available: https:/github.com/timesler/facenetpytorch. [Accessed: 21-Jul-2020].

[49] I. Perov, "GitHub - iperov/DeepFaceLab: DeepFaceLab is the leading software for creating deepfakes." [Online]. Available: https://github.com/iperov/DeepFaceLab. [Accessed: 21-Jul-2020].

[50] "Beijing Internet Court launches online litigation service center." [Online]. Available: https://english.bjinternetcourt.gov.cn/2019-07/01/c_190.htm. [Accessed: 21-Jul-2020].

[51] J.-L. Aufranc, "Lixil AI-based Toilet Analyzes Shape \& Size of Feces with Camera and LEDs." [Online]. Available: https://www.cnx-software.com/2019/10/29/lixil-ai-based-toilet-analyzes-shapesize-of-feces-with-camera-leds/. [Accessed: 21-Jul-2020].

[52] V. Goel and A. Venkataraman, "India's Restaurants Rebel Against Food Delivery Apps - The New York Times." [Online]. Available: https://www.nytimes.com/2019/08/29/technology/india-restaurantslogout-delivery-zomato.html. [Accessed: 21-Jul-2020].

[53] J. Anderer, "Hurry Up! Modern Patience Thresholds Lower Than Ever Before, Survey Finds." [Online]. Available: https://www.studyfinds.org/hurry-up-modern-patience-thresholds-lower-than-everbefore-survey-finds/. [Accessed: 21-Jul-2020]. 